\title{
2D Maps for Visual Analysis and Retrieval in Large Multi-Feature 3D Model Databases
}

\author{
Benjamin Bustos
}

\author{
Daniel A. Keim
}

Christian Panse

Tobias Schreck*

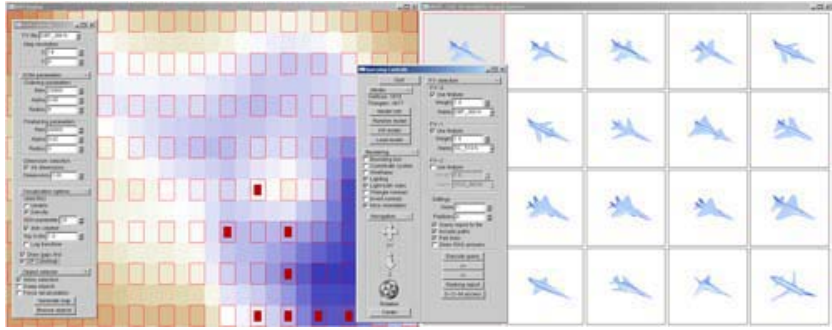

Figure 1: Integrated retrieval and exploration system. By coupling nearest-neighbor querying and SOM visualization, the user can enhance her query by exploring those SOM regions where interesting answer objects are mapped to, possibly finding more relevant objects, which in turn can be used for refining the query using relevance feedback techniques.

- An implementation of more than 15 different state-ofthe-art 3D FT algorithms. For a complete list of FTs in our system, see $\left[\mathrm{BKS}^{+} 04\right]$.

- An interactive browsing and retrieval module, allowing to conduct relevance-feedback supported query-byexample. FTs may be interactively selected and combined by the user for each query.

- An interactive module for generating SOMs from the feature-transformed database, using the SOMPAK implementation from the Helsinki University of Technology [KHKL96]. The SOM rendering subsystem currently includes the U-matrix [Ves99] and the smoothed data histogram techniques [PRM02].

- Tight integration of the SOM and the retrieval module. SOM nodes can be selected by the user, and the objects mapped to the selected nodes can be loaded into the 3D model browser, allowing to explore the SOM's structure. Vice versa, for each retrieved object its respective location on the SOM grid can be highlighted.

- The system is implemented in $\mathrm{C}++$ using the openGL and GLUI libraries, and is running on a MS Windows workstation. Exploration and retrieval are in interactive time, while the SOM generation is done offline.

\section{Application scenarios}

The system supports the following use cases: 

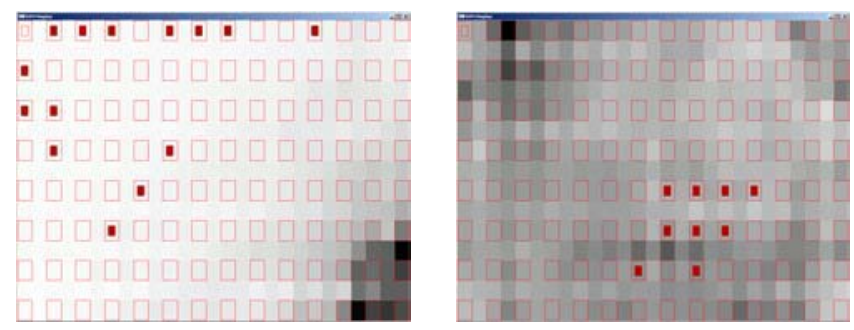

Figure 2: Feature evaluation and selection. SOMs for two different FTs applied to our 3D database. The left and right images show the U-matrix of a moment-based and an imagebased FT, respectively. It can easily be seen that the overall discrimination power of the first FT is much weaker than the discrimination power of the second one, as most nodes have roughly the same low distance between them, which is visualized by bright grey values. Consequently, the members of a class of similar objects (i.e., 26 airplane models) are spread widely over the first map, while they cluster much better on the second map (all nodes containing the airplane objects are labeled red). Comparing U-matrices of different FTs, a user is able to select FTs that are appropriate for performing queries for objects she is interested in.

- The integration of query-by-example driven retrieval and SOM-based overview allows to explore many interesting querying paths simultaneously, as opposed to the linear browsing of $k-N N$ lists returned by standard retrieval systems, c.f. Figure 1.

- U-matrix visualizations are suited to assess the database structure as induced by different FTs, allowing to identify those FTs that suit the user's need, c.f. Figure 2.

- Exploring the SOM structure by browsing objects located at interesting nodes allows the user to quickly gain an overview over the whole database, c.f. Figure 3.

\section{Conclusions}

Preliminary results of our system show the usefulness of the SOM approach for supporting interactive querying and clustering, and for exploring 3D databases using multiple FTs. We sketched how users can be guided in choosing appropriate FTs from a repository of methods at hand.

Future work involves applying the system to organize a database of 25.000 real-world CAD objects we have obtained from a company in the manufacturing industry. The system will also be extended to support media types other than 3D models; currently we are building a feature base for images. As another interesting potential application, we plan to explore the usefulness of SOMs for clustering and classification of large collections of Email, focusing on the identification of characteristics of unsolicited Email traffic.

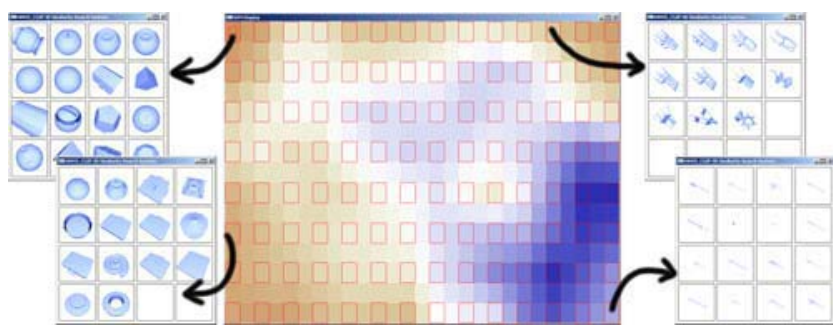

Figure 3: Clustering and overview. The center image displays the allocation frequency of SOM nodes with 3D objects using a smoothed density histogram algorithm, allowing to identify dense clusters on the map (red and blue colors indicate low and high density values, respectively). The surrounding images show four model browser windows displaying objects located at opposing map nodes. The SOM topologically organizes the objects by geometric similarity, discriminating groups of solid-spherical, thinly-prolonged, and roughly planar objects on the corners of the map. Combining both visualizations, a user can quickly gain an overview over large collections of models.

\section{Acknowledgments}

We thank our colleagues Dietmar Saupe and Dejan Vranic for cooperating with us on this project by providing the 3D database, feature extractors, and helpful comments. We also thank Professor Kohonen and his group for providing SOM implementations to the public.

\section{References}

B. Bustos, D. Keim, D. Saupe, T. Schreck, and D. Vranić. Using entropy impurity for improved $3 \mathrm{~d}$ object similarity search. In Proc. IEEE Int. Conf. on Multimedia and Expo (ICME'04), 2004.

T. Kohonen, J. Hynninen, J. Kangas, and J. Laaksonen. Som_pak: The self-organizing map program package. Technical Report A31, Helsinki University of Technology, Laboratory of Computer and Information Science, 1996.

T. Kohonen. Self-Organizing Maps. Springer, Berlin, 3rd edition, 2001.

E. Pampalk, A. Rauber, and D. Merkl. Using smoothed data histograms for cluster visualization in self-organizing maps. In Proc. Int. Conf. on Artifical Neural Networks (ICANN'02), volume 2415 of Lecture Notes in Computer Science. Springer, 2002.

J. Tangelder and R. Veltkamp. A survey of content based 3d shape retrieval methods. In Proc. Shape Modeling International 2004 (SMI'04). IEEE CS Press, 2004.

J. Vesanto. SOM-based data visualization methods. Intelligent Data Analysis, 3(2):111-126, 1999. 